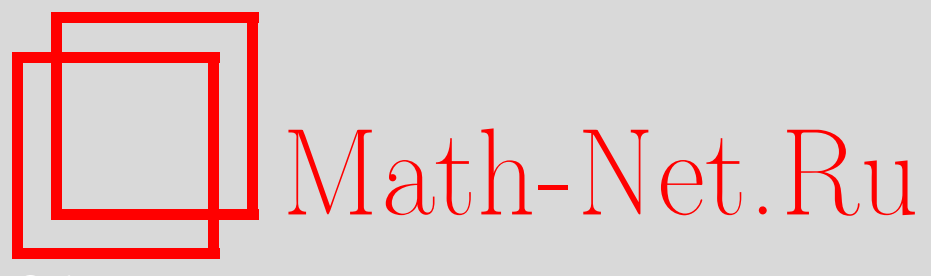

В. П. Маслов, В. Е. Назайкинский, Об одной задаче теории вероятностей, Матем. заметки, 2007, том 81, выпуск 6, 879-892

DOI: https://doi.org/10.4213/mzm3738

Использование Общероссийского математического портала Math-Net.Ru подразумевает, что вы прочитали и согласны с пользовательским соглашением http://www . mathnet.ru/rus/agreement

Параметры загрузки:

IP : 107.22 .136 .117

26 апреля 2023 г., 12:56:30

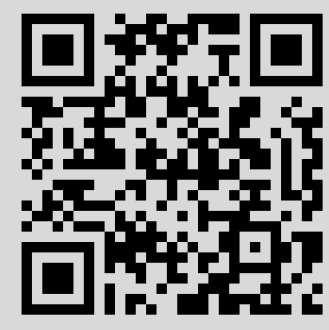


Том 81 выпуск 6 июнь 2007

УДК 519

\section{Об одной задаче теории вероятностей}

\section{В. П. Маслов, В. Е. Назайкинский}

Для непрерывных случайных величин изучается задача, аналогичная рассмотренной ранее одним из авторов для дискретных случайных величин. Заданы числа:

$$
N>0, \quad E>0, \quad 0 \leqslant \lambda_{1} \leqslant \lambda_{2} \leqslant \cdots \leqslant \lambda_{s} .
$$

Рассматривается случайный вектор $x=\left(x_{1}, \ldots, x_{s}\right)$, равномерно распределенный на множестве

$$
x_{j} \geqslant 0, \quad j=1, \ldots, s ; \quad \sum_{j=1}^{s} x_{j}=N, \quad \sum_{j=1}^{s} \lambda_{j} x_{j} \leqslant E .
$$

Исследуется слабый предел $x$ при $s \rightarrow \infty$.

Библиография: 4 названия.

\section{1. Введение и формулировка результатов}

Для непрерывных случайных величин мы изучаем задачу, аналогичную рассмотренной для дискретных случайных величин в [1] (в указанной работе можно также найти дальнейшие ссылки). Пусть

$$
N>0, \quad E>0, \quad 0 \leqslant \lambda_{1} \leqslant \lambda_{2} \leqslant \cdots \leqslant \lambda_{s}
$$

- заданные числа. Рассмотрим случайный вектор $x=\left(x_{1}, \ldots, x_{s}\right)$, равномерно распределенный на множестве

$$
x_{j} \geqslant 0, \quad j=1, \ldots, s ; \quad \sum_{j=1}^{s} x_{j}=N, \quad \sum_{j=1}^{s} \lambda_{j} x_{j} \leqslant E .
$$

Случайные величины $x_{j}$ (которые не являются независимыми, поскольку в $(2)$ имеются связывающие их условия на суммы) можно трактовать как значения случайной функции дискретного аргумента $j \in\{1, \ldots, s\}$. Нас интересует, как устроена эта функция, когда $s$ велико. Оказывается, что (при сформулированных в теореме ниже условиях на параметры (1) рассматриваемой задачи) при большом $s$ она с большой вероятностью близка к детерминированной функции $\bar{x}=\left(\bar{x}_{1}, \ldots, \bar{x}_{s}\right)$, где

$$
\bar{x}_{i}=\frac{1}{\beta \lambda_{i}-\nu}, \quad i=1, \ldots, s,
$$

(C) В.П. МАслов, В.Е. НАЗАЙКинСКий, 2007 
а числа $\beta>0$ и $\nu<0$ определены как решение системы уравнений

$$
\sum_{j=1}^{s} \bar{x}_{j}=N, \quad \sum_{j=1}^{s} \lambda_{j} \bar{x}_{j}=E .
$$

Близость здесь понимается не в смысле нормы $\|x-\bar{x}\|$ (которая может быть с большой вероятностью и не мала), а в следующем более слабом "интегральном" смысле: если $J \subset\{1, \ldots, s\}$ - произвольное фиксированное подмножество, то величина

$$
V(J)=\left|\sum_{j \in J}\left(x_{j}-\bar{x}_{j}\right)\right|
$$

с большой вероятностью мала ${ }^{1}$. (Это свойство можно трактовать как слабую сходимость разности $x-\bar{x}$ к нулю при $s \rightarrow \infty$.)

Перейдем к формулировке результатов. Все доказательства будут даны в 22 . Прежде всего заметим, что система уравнений $(3),(4)$ имеет решение $\beta \geqslant 0, \nu<0$ тогда и только тогда, когда

$$
\frac{N s}{\sum_{j=1}^{s} \lambda_{j}^{-1}} \equiv E_{\min }<E \leqslant E_{\max }=\frac{N}{s} \sum_{j=1}^{s} \lambda_{j},
$$

где левая часть формально полагается равной нулю, если хотя бы одно из чисел $\lambda_{j}$ равно нулю (это утверждение легко проверяется, и мы опустим доказательство). Случай когда $E$ близко к $E_{\min }$, является в некотором смысле вырожденным, и мы будем предполагать, что число $E$ лежит на несколько меньшем отрезке, а именно,

$$
E \in\left[E_{0}, E_{\max }\right]
$$

где $E_{0}=\sum_{j=1}^{s} \lambda_{j}\left(\beta_{0} \lambda_{j}-\nu_{0}\right)^{-1}$, а $\beta_{0}, \nu_{0}$ - такое произвольное решение уравнения $\sum_{j=1}^{s}\left(\beta_{0} \lambda_{j}-\nu_{0}\right)^{-1}=N$, что $N \nu_{0} / s \leqslant-\varkappa, 0 \leqslant N \beta_{0} \lambda_{\max } / s \leqslant \varkappa^{-1}$, а $\varkappa-$ фиксированное произвольно малое число. В дальнейшем через $\beta, \nu, \bar{x}$ обозначим решение системы уравнений $(3),(4)$.

ПРЕДЛОЖЕНИЕ 1. Предположим, что выполнено условие (6) и, кроме того,

(i) либо

$$
\lambda_{1}=\cdots=\lambda_{s}=\lambda \quad\left(\operatorname{ma\kappa } \text { umo } E=\lambda N u \bar{x}_{1}=\cdots=\bar{x}_{s}=N / s\right),
$$

(ii) либо существуют такие постоянные $\alpha \in(0,1] u c_{1}>0$, что

$$
\sum_{j=1}^{s}\left(\frac{(s / N) t-\beta \lambda_{j} \tau}{\beta \lambda_{j}-\nu}\right)^{2} \geqslant c_{1} s^{\alpha}\left(t^{2}+\tau^{2}\right), \quad(t, \tau) \in \mathbb{R}^{2} .
$$

Тогда для произвольных $C_{0}>0, \gamma \in[0,1), \varepsilon \in(0,(1-\gamma) / 2) u \varepsilon_{1}<2 \varepsilon$ существуют такие числа $C, K>0$, не зависящие от $s$, что для любых заданных попарно непересекающихся подмножеств $J_{1}, \ldots, J_{k} \subset\{1, \ldots, s\}$, где $k \leqslant C_{0} s^{\gamma}$, выполнена оценка

$$
\mathbf{P}\left(\sum_{m=1}^{k}\left|\sum_{j \in J_{m}} x_{j}-\sum_{j \in J_{m}} \bar{x}_{j}\right|>\frac{N}{s^{(1-\gamma) / 2-\varepsilon}}\right)<C e^{-K s^{\gamma+\varepsilon_{1}}} .
$$

\footnotetext{
${ }^{1}$ То же самое будет верно и для величины $V\left(J_{1}\right)+\cdots+V\left(J_{k}\right)$, где $J_{1}, \ldots, J_{k}-$ произвольные фиксированные непересекающиеся подмножества в $\{1, \ldots, s\}$, а число $k$ много меньше, чем $s$.
} 
Замечание 1. Неформально условие (ii) означает, что числа $\lambda_{i}$ не сосредоточены в "чрезмерно узком" интервале, в то время как условие (i) дает противоположный крайний случай - все числа $\lambda_{j}$ совпадают, так что минимум выражения в левой части условия (8) на сфере $t^{2}+\tau^{2}=$ const равен нулю. В работе [1] доказана (для задачи с дискретными переменными $x_{i}$ ) без дополнительных предположений (i) или (ii) более грубая, чем (9), оценка (при $\gamma=0$ )

$$
\mathbf{P}\left(\left|\sum_{j \in J} x_{j}-\sum_{j \in J} \bar{x}_{j}\right|>\frac{N}{s^{1 / 4-\varepsilon}}\right)<C e^{-K s^{\varepsilon_{1}}}
$$

(показатель степени в оценке уклонений содержит 1/4 вместо 1/2). Доказательство более тонкой оценки (9) в настоящей работе опирается на исследование двумерных интегралов типа Лапласа. Было бы интересно, комбинируя методы данной работы и [1], распространить эту оценку на общий случай, т.е. избавиться от предположений (i) и (ii).

Сформулируем теперь результаты, вытекающие из предложения 1 для конкретных частных случаев.

Случай, когда все $\lambda_{j}$ одинаковы. Рассмотрим прежде всего случай, когда все $\lambda_{j}$ совпадают. Речь идет о поведении при больших $s$ системы неотрицательных случайных величин $x_{j}, j=1, \ldots, s$, подчиненных единственному условию $\sum_{j=1}^{s} x_{j}=N$ и равномерно распределенных на множестве, задаваемом этим условием. Интерпретируя эти векторы как ступенчатые случайные функции, получаем следующие теоремы о слабом пределе для равномерного распределения с граничными условиями:

Теорема 1. Пусть $f_{s}(t), s=1,2, \ldots,-$ последовательность независимых неотрицательных ступенчатых случайных функиий на интервале $(0,1]$ таких, что $f_{s}(t)$ постоянна на интервалах $t \in((j-1) / s, j / s], j=1, \ldots, s, \int_{0}^{1} f_{s}(t) d t=1 u$ для каждого фиксированного $s$ все функции, удовлетворяющие таким условиям, равновероятны. Тогда при $s \rightarrow \infty$ последовательность $f_{s}(t)$ слабо сходится $\kappa$ детерминированной функиии $f(t)=1$ почти наверное, т.е. для любой интегрируемой по Лебегу пробной функиии $\varphi(t)$

$$
\mathbf{P}\left(\lim _{s \rightarrow \infty} \int_{0}^{1}\left(f_{s}(t)-1\right) \varphi(t) d t=0\right)=1 .
$$

Теорема 2. Пусть $f_{s}(t), s=1,2, \ldots$ - последовательность независимых неубъвающих ступенчатых случайных функций на отрезке $[0,1]$ таких, что $f_{s}(t)$ постоянна на интервалах $t \in((j-1) / s, j / s], j=1, \ldots, s, f_{s}(0)=0, f_{s}(1)=1 u$ для каждого фиксированного s все функции, удовлетворяющие таким условиям, равновероятны. Тогда при $s \rightarrow \infty$ последовательность $f_{s}(t)$ слабо сходится $\kappa$ детерминированной функиии $f(t)=t$ почти наверное, т.е. для любой интегрируемой по Лебегу пробной функиии $\varphi(t)$

$$
\mathbf{P}\left(\lim _{s \rightarrow \infty} \int_{0}^{1}\left(f_{s}(t)-t\right) \varphi(t) d t=0\right)=1 .
$$


Случай, когда $\lambda_{j}$ различны. Пусть задана последовательность

$$
0 \leqslant \lambda_{1}<\lambda_{2}<\cdots<\lambda_{j}<\cdots
$$

попарно различных неотрицательных чисел, причем эта последовательность возрастает равномерно том смысле, что

$$
\lambda_{j} \simeq \text { const } \cdot j \quad \text { при } \quad j \rightarrow \infty .
$$

(Примером такой последовательности может служить $\lambda_{j}=j$ или $\lambda_{j}=j+a j^{-b}$, где $a, b>0$ и $a$ достаточно мало́, так что последовательность возрастает при всех $j$.) Для заданного $s=1,2, \ldots$ "обрежем" последовательность (11) на $s$-м члене, т.е. оставим только ее начальный отрезок $\lambda_{1}, \ldots, \lambda_{s}$, и рассмотрим случайный вектор $x=\left(x_{1}, \ldots, x_{s}\right)$, равномерно распределенный на множестве $(2)$. Поведение таких случайных величин при $s \rightarrow \infty$ описывается следующей теоремой.

ТЕОРема 3. При выполнении условий (11), (12) и условия (6) для Е для произвольных $C_{0}, \beta_{\min }>0, \mu \in[0,1 / 2), \gamma \in[0,1), \varepsilon \in(0,(1-\gamma) / 2) u \varepsilon_{1}<2 \varepsilon$ существуют такие числа $C, K>0$, не зависящие от $s$, что для любых заданных попарно непересекающихся подмножеств $J_{1}, \ldots, J_{k} \subset\{1, \ldots, s\}$, где $k \leqslant C_{0} s^{\gamma}$, справедлива оченка (9) при условии, что

$$
\beta>\beta_{\min } s^{-\mu} N^{-1} \text {. }
$$

ПримеР 1 (см. также [4]). Рассмотрим задачу об безрисковом инвестировании ограниченного объема капиталовложений в инвестиционные фонды из заданного списка с заданной ранжировкой по приоритетности. Безрисковость будем понимать в смысле гипотезы "zero intelligence" для финансовых рынков: действия трейдеров по покупке и продаже акиий можно считать (псевдо)случайными. Тогда все варианты распределения вложений при выполнении заданных ограничений равноправны, и если найдется такое распределение вложений, что большинство возможных вариантов распределения находится вблизи него, то оно и будет безрисковым.

Пусть задан список из $s$ инвестиционных фондов $A_{1}, \ldots, A_{s}$. Предполагается, что сумма $C_{i}$ инвестиций в $i$-й инвестиционный фонд может быть произвольна. В такой ситуации разумно считать ее непрерывной переменной. Пусть заданы ограничения

$$
\begin{gathered}
\sum_{j=1}^{s} C_{j} \leqslant \Phi \quad \text { (бюджетное ограничение) }, \\
K=C_{1} \leqslant C_{2} \leqslant \cdots \leqslant C_{s}=M \quad \text { (условие приоритетности). }
\end{gathered}
$$

Если обозначить

$$
x_{j}=C_{s-j+1}-C_{s-j} \geqslant 0, \quad j=1, \ldots, s-1,
$$

то

$$
\begin{array}{r}
\sum_{j=1}^{s-1} x_{j}=M-K \equiv N, \\
\sum_{j=1}^{s-1} j x_{j} \leqslant \Phi^{\prime}=\Phi-K s \equiv E .
\end{array}
$$


Таким образом, в данной задаче $\lambda_{j}=j$ и можно применить теорему 3 . Получаем, что (при выполнении соответствующих условий на $E$ и $\beta$ ) доля числа вариантов, для которых при заданном $l$

$$
\left|C_{l}-C_{1}-\sum_{j=s-l+1}^{s-1} \frac{1}{\beta j-\nu}\right|>\frac{N}{s^{1 / 2-\varepsilon}},
$$

где $\varepsilon>0$, не превосходит $c_{1} e^{-s^{\delta}}, \delta=\delta(\varepsilon)>0$. Итак, "безрисковое" вложение задается формулами

$$
C_{i}=K+\sum_{j=s-l+1}^{s-1} \frac{1}{\beta j-\nu}, \quad i=1, \ldots, s .
$$

\section{2. Доказательства предложения и теорем}

Будем в дальнейшем использовать обозначения

$$
\begin{gathered}
\mathscr{N}(x)=\sum_{i=1}^{s} x_{i}, \quad \mathscr{E}(x)=\sum_{i=1}^{s} \lambda_{i} x_{i}, \quad x \in \mathbb{R}^{s}, \\
\Delta_{s-1}=\left\{x \in \mathbb{R}^{s}: x_{i} \geqslant 0, i=1, \ldots, s ; \sum_{j=1}^{s} x_{i}=s\right\} .
\end{gathered}
$$

ДОКАЗАТЕЛЬСТво ПРЕДЛОЖЕНИЯ 1. Прежде всего заметим, что без ограничения общности можно считать, что

$$
N=s, \quad 0 \leqslant \lambda_{i} \leqslant 1, \quad i=1, \ldots s,
$$

что и будет предполагаться в дальнейшем. Действительно, этого можно добиться преобразованием

$$
\begin{gathered}
N \rightarrow s, \quad E \rightarrow \frac{s E}{N \lambda_{s}}, \quad \lambda_{j} \rightarrow \frac{\lambda_{j}}{\lambda_{s}}, \quad x \rightarrow \frac{s}{N} x, \\
\beta \rightarrow \frac{N \lambda_{s}}{s} \beta, \quad \nu \rightarrow \frac{N}{s} \nu, \quad \bar{x} \rightarrow \frac{s}{N} \bar{x},
\end{gathered}
$$

относительно которого все формулы, предположения и утверждения, используемые в предложении, инвариантны. При этом оценка в утверждении предложения приобретает вид

$$
\mathbf{P}\left(\sum_{m=1}^{k}\left|\sum_{j \in J_{m}} x_{j}-\sum_{j \in J_{m}} \bar{x}_{j}\right|>s^{(1+\gamma) / 2+\varepsilon}\right)<C e^{-K s^{\gamma+\varepsilon_{1}}},
$$

а оценка в условии (ii) - вид

$$
\sum_{j=1}^{s}\left(\frac{t-\beta \lambda_{j} \tau}{\beta \lambda_{j}-\nu}\right)^{2} \geqslant c_{1} s^{\alpha}\left(t^{2}+\tau^{2}\right), \quad(t, \tau) \in \mathbb{R}^{2} .
$$

Разберем последовательно указанные в предложении случаи. 
Пусть выполнено условие (i). Тогда $\bar{x}_{j}=1, j=1, \ldots, s$. Будем считать во избежание громоздкости, что $k=1$, а в конце доказательства укажем изменения, которые нужно сделать в общем случае. Имеем

$$
\begin{aligned}
\mathbf{P}\left(\left|\sum_{j \in J} x_{j}-\sum_{j \in J} 1\right|>\delta\right) & =\frac{1}{\operatorname{vol} \Delta_{s-1}} \int_{A(\delta)} d x_{1} \ldots d x_{s-1} \\
& =\frac{1}{\operatorname{vol} \Delta_{s-1}} \int_{B(\delta)} d x_{1} \ldots d x_{s-1} d x_{s}
\end{aligned}
$$

где

$$
\begin{aligned}
& A(\delta)=\left\{x \in \Delta_{s-1}:\left|\sum_{j \in J} x_{j}-\sum_{j \in J} 1\right|>\delta\right\}, \\
& B(\delta)=\left\{x \in \mathbb{R}^{s}: x=y+(0, \ldots, 0, \tau), y \in A(\delta), \tau \in[0,1]\right\} .
\end{aligned}
$$

Обозначим

$$
\phi(x)=\sum_{j \in J}\left(x_{j}-1\right)
$$

На множестве $B(\delta)$ для любого $c>0$ справедливо неравенство

$$
1 \leqslant e^{s+2-c \delta} e^{-\sum_{j=1}^{s} x_{j}} e^{c|\phi(x)|} \leqslant e^{s+2-c \delta} e^{-\sum_{j=1}^{s} x_{j}}\left(e^{c \phi(x)}+e^{-c \phi(x)}\right)
$$

Поэтому

$$
\begin{aligned}
\int_{B(\delta)} d x_{1} \ldots d x_{s} & \leqslant e^{s+2-c \delta} \int_{B(\delta)} e^{-\sum_{j=1}^{s} x_{j}}\left(e^{c \phi(x)}+e^{-c \phi(x)}\right) d x_{1} \ldots d x_{s} \\
& \leqslant e^{s+2-c \delta} \int_{\mathbb{R}_{+}^{s}} e^{-\sum_{j=1}^{s} x_{j}}\left(e^{c \phi(x)}+e^{-c \phi(x)}\right) d x_{1} \ldots d x_{s} \\
& \equiv e^{s+2-c \delta}\left(I_{+}+I_{-}\right)
\end{aligned}
$$

Пусть $l=\#(J)$ - число элементов во множестве $J$. Тогда

$$
I_{ \pm}=\exp \{-l(\ln (1 \mp c) \pm c)\}=\exp \left\{\frac{l c^{2}}{2(1+\theta c)}\right\} \leqslant \exp \left\{\frac{s c^{2}}{2(1+\theta c)}\right\}
$$

где $|\theta| \leqslant 1$, и, значит, при $0 \leqslant c \leqslant 1 / 2$ и достаточно больших $s$ таких, что

$$
\operatorname{vol} \Delta_{s-1}=\frac{s^{s-1}}{(s-1) !} \geqslant \frac{2}{3} \frac{e^{s}}{\sqrt{2 \pi s}},
$$

имеем

$$
\mathbf{P}\left(\left|\sum_{j \in J} x_{j}-\sum_{j \in J} 1\right|>\delta\right) \leqslant \frac{3 \sqrt{2 \pi s}}{2 e^{s}} \cdot 2 e^{s+2-c \delta+s c^{2}}=3 \sqrt{2 \pi s} e^{2-c \delta+s c^{2}} .
$$


Взяв $\delta=s^{1 / 2+\varepsilon}$ и минимизируя правую часть по $c$ (минимум достигается в точке $\left.\delta /(2 s)=s^{-1 / 2+\varepsilon} / 2 \in[0,1 / 2]\right)$, получаем

$$
\mathbf{P}\left(\left|\sum_{j \in J} x_{j}-\sum_{j \in J} 1\right|>s^{1 / 2+\varepsilon}\right) \leqslant 3 \sqrt{2 \pi s} e^{2-s^{2 \varepsilon} / 4} \leqslant C e^{-K s^{2 \varepsilon}}
$$

для любого $K<1 / 4$ при достаточно большом $C$ (зависящем от $K)^{2}$.

Укажем изменения, необходимые в доказательстве при произвольном $k \leqslant C_{0} s^{\gamma}$. Вместо функции $\phi(x)$ вводятся функции

$$
\phi_{m}(x)=\sum_{j \in J_{m}}\left(x_{j}-1\right), \quad m=1, \ldots, k ;
$$

тогда в формулах $(18)$ и (19) сумма $e^{c \phi(x)}+e^{-c \phi(x)}$ заменяется на произведение

$$
\prod_{m=1}^{k}\left(e^{c \phi_{m}(x)}+e^{-c \phi_{m}(x)}\right)
$$

а в правой части оценки (2) сумма $I_{+}+I_{-}$заменяется на сумму $2^{k}$ членов, каждый из которых отвечает одному из слагаемых в сумме, получающейся, если раскрыть скобки в произведении (21). При $0 \leqslant c \leqslant 1 / 2$ каждый из этих членов по-прежнему оценивается через $e^{s c^{2}}$. С учетом того, что число их не превосходит $2^{C_{0} s^{\gamma}}=e^{C_{0} s^{\gamma} \ln 2}$, аналогично (20) получаем

$$
\mathbf{P} \leqslant \frac{3}{2} \sqrt{2 \pi s} e^{2+C_{0} s^{\gamma} \ln 2-c \delta+s c^{2}} .
$$

Взяв $\delta=s^{(\gamma+1) / 2+\varepsilon}$ и минимизируя правую часть по $c$, получаем

$$
\mathbf{P} \leqslant \frac{3}{2} \sqrt{2 \pi s} e^{2+C s^{\gamma} \ln 2-s^{\gamma+2 \varepsilon} / 4}
$$

откуда немедленно следует нужная оценка.

Пусть выполнено условие (ii). Заметим, что из неравенств (6) вытекает, что при некоторых $b, \beta_{0}, c_{0}>0$, не зависящих от $s$, выполнены неравенства

$$
0<\beta \leqslant \beta_{0}, \quad \nu<-c_{0}, \quad 1 / b<\bar{x}_{i} \leqslant b, \quad i=1, \ldots, s,
$$

которые будут использованы в дальнейшем.

Мы опять считаем, что $k=1$; необходимые изменения для общего $k$ аналогичны указанным в п. (i). Обозначим

$$
\begin{gathered}
\phi(x)=\sum_{j \in J}\left(x_{j}-\bar{x}_{j}\right), \\
D_{\delta}=\left\{x \in \Delta_{s-1}: \mathscr{E}(x) \leqslant E,|\phi(x)| \geqslant \delta\right\}, \\
\Gamma(E, s, \delta)=\int_{D_{\delta}} d x_{1} \ldots d x_{s-1}, \quad \Gamma(E, s)=\Gamma(E, s, 0) .
\end{gathered}
$$

${ }^{2}$ Полагая в конце доказательства $\delta=s^{1 / 2} \ln s$, можно также получить оценку

$$
\mathbf{P}\left(\left|\sum_{j \in J} x_{j}-\sum_{j \in J} 1\right|>s^{1 / 2} \ln s\right) \leqslant C s^{-K \ln s} \leqslant C_{m} s^{-m}, \quad m=1,2, \ldots,
$$

с некоторыми постоянными $C_{m}$. 
Вероятность, которую нужно оценить (обозначим ее просто через $\mathbf{P}$ ) есть отношение

$$
\mathbf{P}=\frac{\Gamma\left(E, s, s^{1 / 2+\varepsilon}\right)}{\Gamma(E, s)} .
$$

Мы получим оценку сверху для $\Gamma(E, s, \delta)$ и оценку снизу для $\Gamma(E, s)$, что и даст нужную оценку для $\mathbf{P}$.

Оценка сверху для меры множества вариантов с большими уклонениями. Эта оценка по существу доказывается так же, как и в случае (i) (оценка (ii) при этом не используется). Справедливо соотношение

$$
\Gamma(E, s, \delta)=\int_{\widetilde{D}_{\delta}} d x_{1} \ldots d x_{s},
$$

где

$$
\widetilde{D}_{\delta}=\left\{x \in \mathbb{R}^{s}: x=y+(\tau, 0, \ldots, 0,), y \in D_{\delta}, \tau \in[0,1]\right\} .
$$

На множестве $\widetilde{D}_{\delta}$ справедливы неравенства

$$
\mathscr{N}(x) \leqslant s+1, \quad \mathscr{E}(x) \leqslant E+\lambda_{1}, \quad|\phi(x)| \geqslant \delta-1,
$$

а следовательно, для любого $c \geqslant 0$, неравенство

$$
\begin{aligned}
1 & \leqslant e^{\beta E+\beta \lambda_{1}-\nu s-\nu-c \delta+c} e^{-\beta \mathscr{E}(x)+\nu \mathscr{N}(x)} e^{c|\phi(x)|} \\
& \leqslant e^{\beta E-\nu s+\beta \lambda_{1}-\nu+c-c \delta} e^{-\beta \mathscr{E}(x)+\nu \mathscr{N}(x)}\left(e^{c \phi(x)}+e^{-c \phi(x)}\right) .
\end{aligned}
$$

Кроме того, функция в правой части (24) всюду положительна. Поэтому, заменяя подынтегральное выражение в (23) на эту функцию и расширяя область интегрирования до положительного октанта $\mathbb{R}_{+}^{s}$, получаем оценку

$$
\Gamma(E, s, \delta) \leqslant e^{c+\beta \lambda_{1}-\nu} e^{\beta E-\nu s-c \delta}\left(I_{+}+I_{-}\right),
$$

где

$$
I_{ \pm}=\int_{\mathbb{R}_{+}^{s}} e^{-\beta \mathscr{E}(x)+\nu \mathscr{N}(x) \pm c \phi(x)} d x_{1} \ldots d x_{s} .
$$

Оценим, например, интеграл $I_{+}\left(I_{-}\right.$оценивается точно так же). Имеем

$$
I_{+}=\prod_{j \in J} e^{-c \bar{x}_{i}-\ln \left(\beta \lambda_{j}-\nu-c\right)} \prod_{j \notin J} e^{-\ln \left(\beta \lambda_{j}-\nu-c\right)}
$$

Далее, по формуле Тейлора с остаточным членом при $0 \leqslant c \leqslant|\nu| / 2$ имеем

$$
\begin{aligned}
-c \bar{x}_{i}-\ln \left(\beta \lambda_{j}-\nu-c\right) & =-\ln \left(\beta \lambda_{j}-\nu\right)+\frac{c}{\beta \lambda_{j}-\nu}+\frac{c^{2}}{2}\left(\frac{1}{\beta \lambda_{j}-\nu-\theta c}\right)^{2}-c \bar{x}_{i} \\
& =-\ln \left(\beta \lambda_{j}-\nu\right)+\frac{c^{2}}{2}\left(\frac{1}{\beta \lambda_{j}-\nu-\theta c}\right)^{2} \\
& \leqslant-\ln \left(\beta \lambda_{j}-\nu\right)+\frac{c^{2}}{2}\left(\frac{1}{\beta \lambda_{j}-\nu / 2}\right)^{2} \\
& \leqslant-\ln \left(\beta \lambda_{j}-\nu\right)+\frac{c^{2}}{2} \cdot 4\left(\frac{1}{\beta \lambda_{j}-\nu}\right)^{2}
\end{aligned}
$$


для некоторого $\theta \in[0,1]$, так что

$$
\Gamma(E, s, \delta) \leqslant 2 e^{c+\beta \lambda_{1}-\nu} e^{\beta E-\nu s-\sum_{j=1}^{s} \ln \left(\beta \lambda_{j}-\nu\right)} e^{-c \delta+K_{1} s c^{2} / 2},
$$

где $K_{1}$ - такая постоянная (не зависящая от $s$ ), что

$$
\sum_{j=1}^{s}\left(\frac{1}{\beta \lambda_{j}-\nu}\right)^{2}<\frac{K_{1} s}{4}
$$

Полагая $\delta=s^{1 / 2+\varepsilon}$ и минимизируя правую часть по $c$ (минимум достигается в точке $c=\delta /(K s)=K^{-1} s^{-1 / 2+\varepsilon} ;$ при достаточно больших $s$ эта величина меньше, чем $c_{0} / 2$, так что в силу второго условия из (22) эта точка действительно лежит на отрезке $[0,|\nu| / 2])$, получаем окончательную верхнюю оценку

$$
\Gamma\left(E, s, s^{1 / 2+\varepsilon}\right) \leqslant \text { const } e^{\beta E-\nu s-\sum_{i=1}^{s} \ln \left(\beta \lambda_{j}-\nu\right)} e^{-s^{2 \varepsilon} /\left(2 K_{1}\right)} .
$$

(Здесь постоянной const мы оценили сомножитель $2 e^{c+\beta \lambda_{1}-\nu}$.)

Оценка снизу для меры множества всех вариантов. Для $\Gamma(E, s)$ справедлива формула

$$
\begin{aligned}
\Gamma(E, s) & =\int_{\Delta_{s-1}} \Theta(E-\mathscr{E}(x)) d x_{1} \ldots d x_{s-1} \\
& =\int_{\mathbb{R}_{+}^{s}} \Theta(E-\mathscr{E}(x)) \delta(\mathscr{N}(x)-s) d x_{1} \ldots d x_{s} .
\end{aligned}
$$

(Заметим, что произведение $\delta$ - и $\Theta$-функций корректно определено, поскольку в силу условия (8) не все $\lambda_{i}$ одинаковы, а значит, градиенты функций $\mathscr{N}(x)$ и $\mathscr{E}(x)$ линейно независимы.) Воспользуемся интегральными представлениями $\Theta$ - и $\delta$-функции

$$
\begin{aligned}
\delta(\mathscr{N}(x)-s) & =\lim _{a \rightarrow 0} \frac{e^{\nu(\mathscr{N}(x)-s)}}{2 \pi} \int_{-\infty}^{\infty} e^{i t(\mathscr{N}(x)-s)} \rho(a t) d t \\
\Theta(y) & =\frac{1}{2 \pi i} \lim _{a \rightarrow 0} \int_{-\infty}^{\infty} \frac{e^{\beta y(1+i \tau)}}{\tau-i} \rho(a \tau) d \tau
\end{aligned}
$$

(предел в смысле *-слабой сходимости линейных функционалов), где $\rho$ - финитная функция, равная единице в начале координат, подставим их в формулу (26) и проведем интегрирование по всем $x_{i}$ от 0 до $\infty$; затем, переходя в подынтегральном выражении к пределу при $a \rightarrow 0$, получим

$$
\Gamma(E, s)=\frac{e^{\beta E-\nu N-\sum_{j=1}^{s} \ln \left(\beta \lambda_{j}-\nu\right)}}{4 \pi^{2} i} \iint_{\mathbb{R}^{2}} \frac{e^{s \Phi(t, \tau)}}{\tau-i} d \tau d t,
$$

где

$$
\Phi(t, \tau)=i \frac{\beta E}{s} \tau-i t-\frac{1}{s} \sum_{j=1}^{s}\left[\ln \left(\beta \lambda_{j}-\nu+i \beta \lambda_{j} \tau-i t\right)-\ln \left(\beta \lambda_{j}-\nu\right)\right],
$$

а аргумент выражения под знаком логарифма выбирается в интервале $(-\pi / 2, \pi / 2)$. (Как мы увидим ниже, интеграл (27) абсолютно сходится, так что переход к пределу в подынтегральном выражении - законная процедура.) 
Дадим оценку снизу интеграла (27), используя метод, аналогичный применявшемуся в статье [3]. Дополнительная сложность (по сравнению со случаем, который рассматривался в указанной статье) состоит в том, что фазовая функция комплексна и, кроме того, сама зависит от большого параметра. Однако ее специальная структура позволяет тем не менее провести нужные оценки.

Обозначим

$$
d_{j} \equiv d_{j}(t, \tau)=\frac{\beta \lambda_{j} \tau-t}{\beta \lambda_{j}-\nu}, \quad j=1, \ldots, s, \quad r=\sqrt{t^{2}+\tau^{2}} .
$$

Тогда, используя уравнения (1), получаем

$$
\begin{aligned}
\Phi(t, \tau) & =i \frac{\beta E}{s} \tau-i t-\frac{1}{s} \sum_{j=1}^{s} \ln \left[1+i d_{j}(t, \tau)\right] \\
& =\frac{1}{s} \sum_{j=1}^{s}\left(-\ln \left[1+d_{j}^{2}\right]+i\left[d_{j}-\arctan d_{j}\right]\right) .
\end{aligned}
$$

Из наложенных нами условий вытекает, что все производные функции $\Phi$ равномерно по $s$ ограничены. Кроме того,

$$
\Phi(0,0)=0, \quad \operatorname{Re} \Phi(t, \tau) \leqslant 0 .
$$

Разобьем интеграл (27) на два интеграла:

$$
\begin{aligned}
\Gamma(E, s) & =\frac{e^{\beta E-\nu N-\sum_{j=1}^{s} \ln \left(\beta \lambda_{j}-\nu\right)}}{4 \pi^{2}}\left(\frac{1}{i} \int_{r \leqslant 1}(\ldots)+\frac{1}{i} \int_{r \geqslant 1}(\ldots)\right) \\
& \equiv \frac{e^{\beta E-\nu N-\sum_{j=1}^{s} \ln \left(\beta \lambda_{j}-\nu\right)}}{4 \pi^{2}}\left(I_{1}+I_{2}\right)
\end{aligned}
$$

и изучим каждый из них по отдельности. Интеграл (27) вещественный, поэтому при достаточно оценить вещественные части интегралов $I_{1}$ и $I_{2}$. Прежде всего займемся интегралом

$$
I_{1}=\iint_{r<1} e^{s \operatorname{Re} \Phi} \frac{e^{i s \operatorname{Im} \Phi}}{1+i \tau} d \tau d t \equiv \iint_{r<1} e^{s \operatorname{Re} \Phi} f(s, t, \tau) d \tau d t .
$$

В круге $\{r \leqslant 1\}$ справедливы неравенства

$$
\left|d_{j}\right| \leqslant C_{1}, \quad \frac{C_{2}}{s} \sum_{j=1}^{s} d_{j}^{2} \geqslant-\operatorname{Re} \Phi \geqslant \frac{C_{3}}{s} \sum_{j=1}^{s} d_{j}^{2}, \quad|\operatorname{Im} \Phi| \leqslant \frac{C_{4}}{s} \sum_{j=1}^{s} d_{j}^{3}
$$

(здесь и ниже $C_{j}$ - положительные постоянные, на зависящие от $s$ ). Далее, с учетом (17) имеем

$$
c_{1} s^{\alpha} r^{2} \leqslant \sum_{j=1}^{s} d_{j}^{2} \leqslant C_{1}^{2} s r^{2}
$$


Поэтому

$$
\sum_{j=1}^{s} d_{j}^{3} \leqslant C_{1} r \sum_{j=1}^{s} d_{j}^{2} \leqslant C_{1} r \sum_{j=1}^{s} d_{j}^{2} \frac{\left(\sum_{j=1}^{s} d_{j}^{2}\right)^{1 / 2}}{c_{1}^{1 / 2} s^{\alpha / 2} r}=C_{5}\left(\sum_{j=1}^{s} d_{j}^{2}\right)^{3 / 2} s^{-\alpha / 2} .
$$

Комбинируя эту оценку с (30), получаем, что в круге $\{r \leqslant 1\}$ справедливо неравенство

$$
|\operatorname{Im} \Phi| \leqslant C_{6} s^{(1-\alpha) / 2}(-\operatorname{Re} \Phi)^{3 / 2} .
$$

Разобьем круг $D=\{r \leqslant 1\}$ на три подмножества:

$$
D=A \cup(B \backslash A) \cup C,
$$

где

$$
\begin{aligned}
& A=\left\{(t, \tau) \in D: r \leqslant a s^{-1 / 2}\right\} \\
& B=\left\{(t, \tau) \in D:-\operatorname{Re} \Phi \leqslant b s^{\alpha / 3-1}\right\}, \\
& C=\left\{(t, \tau) \in D:-\operatorname{Re} \Phi>b s^{\alpha / 3-1}\right\},
\end{aligned}
$$

где $a$ и $b$ - некоторые постоянные, не зависящие от $s$. На множестве $B$ выполнено условие

$$
|s \operatorname{Im} \Phi| \leqslant C_{6} s \cdot s^{(1-\alpha) / 2}\left(b s^{\alpha / 3-1}\right)^{3 / 2}=C_{6} b^{3 / 2} .
$$

Поэтому для достаточно малого $b$ аргумент функции $f(s, t, \tau)$ при $(t, \tau) \in B$ будет лежать в интервале $\left(-\pi / 2+C_{7}, \pi / 2-C_{7}\right)$, а так как ее модуль не меньше $1 / \sqrt{2}$, то на множестве $B$ справедливо неравенство

$$
\operatorname{Re} f(s, t, \tau)>C_{8}
$$

Итак, вещественная часть подынтегрального выражения на множестве $B$ будет положительна. Далее, на множестве $A$ справедливо неравенство

$$
-\operatorname{Re} \Phi \leqslant C_{9} a^{2} s^{-1}
$$

поэтому при достаточно малом а справедливо включение $A \subset B$ и, следовательно, вещественная часть интеграла по $B$ оценивается снизу через вещественную часть интеграла по $A$. На множестве $A$ вещественная часть подынтегрального выражения не меньше, чем $C_{8} e^{-C_{9} a^{2}}$, а его площадь равна $\pi a^{2} s^{-1}$, так что в итоге получаем, что интеграл по множеству $A$, а значит, и по множеству $B$, оценивается снизу величиной $C_{10} s^{-1}$. Далее, на множестве $C$ вещественная часть показателя экспоненты удовлетворяет неравенству

$$
s \operatorname{Re} \Phi \leqslant-b s^{\alpha / 3},
$$

так что этот интеграл экспоненциально мал. Окончательно получаем

$$
\operatorname{Re} I_{1} \geqslant C_{11} s^{-1}
$$


при больших $s$. Оценим теперь интеграл $I_{2}$ :

$$
\begin{aligned}
\left|I_{2}\right| & \leqslant \iint_{r>1} \frac{e^{s \operatorname{Re} \Phi}}{\sqrt{1+\tau^{2}}} d \tau d t \\
& =\iint_{r>1} \frac{e^{s \operatorname{Re} \Phi+\ln \left(1+r^{2} / c_{1}\right)}}{\left(1+r^{2} / c_{1}\right) \sqrt{1+\tau^{2}}} d \tau d t \\
& \leqslant C_{12} \exp \left\{\sup _{r>1}\left[s \operatorname{Re} \Phi+\ln \left(1+\frac{r^{2}}{c_{1}}\right)\right]\right\},
\end{aligned}
$$

где

$$
C_{12}=\iint \frac{1}{\left(1+r^{2} / c_{1}\right) \sqrt{1+\tau^{2}}} d \tau d t<\infty
$$

а постоянная $c_{1}>0$ та же, что и в (17). Оценим супремум в (33). Пусть

$$
d^{2}=\sum_{j=1}^{s} d_{j}^{2}, \quad \alpha_{j}=d_{j}^{2} / d^{2}, \quad j=1, \ldots, s .
$$

Тогда

$$
s \operatorname{Re} \Phi+\ln \left(1+\frac{r^{2}}{c_{1}}\right) \leqslant s \operatorname{Re} \Phi+\sum_{j=1}^{s} \ln \left(1+\frac{\alpha_{j} r^{2}}{c_{1}}\right)=-\sum_{j=1}^{s} \ln \left(\frac{1+d_{j}^{2}}{1+d_{j}^{2} r^{2} /\left(c_{1} d^{2}\right)}\right) .
$$

Согласно (17) имеем $r^{2} / d^{2} \leqslant c_{1} s^{-\alpha}$, поэтому

$$
\ln \left(\frac{1+d_{j}^{2}}{1+d_{j}^{2} r^{2} /\left(c_{1} d^{2}\right)}\right) \geqslant \ln \left(\frac{1+d_{j}^{2}}{1+d_{j}^{2} s^{-\alpha}}\right)=\int_{1+d_{j}^{2} s^{-\alpha}}^{1+d_{j}^{2}} \frac{d x}{x} \geqslant\left(1-s^{-\alpha}\right) \frac{d_{j}^{2}}{1+d_{j}^{2}}
$$

и

$$
s \operatorname{Re} \Phi+\ln \left(1+\frac{r^{2}}{c_{1}}\right) \leqslant-\left(1-s^{-\alpha}\right) \sum_{j=1}^{s} \frac{d_{j}^{2}}{1+d_{j}^{2}} .
$$

Функция $d_{j}^{2} /\left(1+d_{j}^{2}\right)$ возрастает с ростом $r$, поэтому правая часть не превышает своего значения на сфере $r=1$, а оно в свою очередь не превышает $-C_{13} \sum_{j=1}^{s} d_{j}^{2} \geqslant$ $-C_{14} s^{\alpha}$. Итак, интеграл $I_{2}$ также экспоненциально мал, и в итоге получаем для $\Gamma(E, s)$ оценку

$$
\Gamma(E, s) \geqslant C_{15} \frac{e^{\beta E-\nu N-\sum_{j=1}^{s} \ln \left(\beta \lambda_{j}-\nu\right)}}{s} .
$$

Комбинируя эту оценку с оценкой (25), получаем утверждение предложения.

ДоКАЗАТЕЛЬСТво тЕОРЕм 1 и 2. Докажем теорему 1 (вывод теоремы 2 аналогичен). Функциям $f_{s}$ можно взаимно однозначно сопоставить векторы $x$ из предложения 1 по правилу

$$
f_{s}(t)=x_{j} \quad \text { при } t \in((j-1) / s, j / s], \quad j=1, \ldots, s .
$$

Достаточно доказать утверждение для случая, когда $\varphi$ - характеристическая функция некоторого отрезка $[a, b] \subset[0,1]$. Для каждого $s$ этот отрезок покрывается 
отрезками $X_{j}=[(j-1) / s, j / s], j=m_{1}, \ldots, m_{2}$, (где $m_{1}, m_{2}$ зависят от $\left.s\right)$, так что $a \in X_{m_{1}}, b \in X_{m_{2}}$,

$$
\bigcup_{j=m_{1}+1}^{m_{2}-1} X_{j} \subseteq[a, b] \subseteq \bigcup_{j=m_{1}}^{m_{2}} X_{j}
$$

Поэтому

$$
\left|\int_{0}^{1}\left(f_{s}(t)-1\right) \varphi(t) d t\right| \leqslant \frac{1}{s}\left(\left|\sum_{j \in J_{s 1}}\left(x_{j}-1\right)\right|+\left|\sum_{j \in J_{s 2}}\left(x_{j}-1\right)\right|+\left|\sum_{j \in J_{s 3}}\left(x_{j}-1\right)\right|\right),
$$

где $J_{s 1}=\left\{m_{1}\right\}, J_{s 2}=\left\{m_{1}+1, \ldots, m_{2}-1\right\}, J_{s 3}=\left\{m_{2}\right\}$. Обозначим сумму в правой части равенства (4) через $z_{s}$. Достаточно доказать, что $z_{s} \rightarrow 0$ п.н. Согласно предложению 1

$$
\mathbf{P}\left(z_{s}>s^{-1 / 2+\varepsilon}\right)<C e^{-K s^{\varepsilon_{1}}},
$$

поэтому для любого $\delta>0$ при достаточно больших $s$

$$
\mathbf{P}\left(z_{s}>\delta\right)<C e^{-K s^{\varepsilon_{1}}}, \quad \mathbf{P}\left(z_{s} \leqslant \delta\right)>1-C e^{-K s^{\varepsilon_{1}}} .
$$

Далее, обозначим через $A_{k \delta}$ событие

$$
A_{k \delta}=\left\{z_{s} \leqslant \delta \text { для всех } s \geqslant k\right\} .
$$

Тогда для любого достаточно большого $k \geqslant k(\delta)$ имеем

$$
\mathbf{P}\left(A_{k \delta}\right) \geqslant \prod_{s \geqslant k}\left(1-C e^{-K s^{\varepsilon_{1}}}\right) \geqslant 1-C \sum_{s \geqslant k} e^{-K s^{\varepsilon_{1}}} \rightarrow 1
$$

при $k \rightarrow \infty$ и фиксированном $\delta$. Вычислим теперь вероятность сходимости $z_{s} \rightarrow 0$. Имеем

$$
\mathbf{P}\left(z_{s} \rightarrow 0\right)=\mathbf{P}\left(\forall \delta>0 \exists k \forall s \geqslant k: z_{s} \leqslant \delta\right)=\mathbf{P}\left(\bigcap_{\delta} \bigcup_{k} A_{k \delta}\right)=\mathbf{P}\left(\bigcap_{\delta} B_{\delta}\right),
$$

где $B_{\delta}=\bigcup_{k} A_{k \delta}$. Поскольку $A_{k \delta} \subset A_{k^{\prime} \delta}$ при $k \leqslant k^{\prime}$ и $B_{\delta} \subset B_{\delta^{\prime}}$ при $\delta \geqslant \delta^{\prime}$, то

$$
\mathbf{P}\left(B_{\delta}\right)=\lim _{k \rightarrow \infty} \mathbf{P}\left(A_{k \delta}\right)=1, \quad \mathbf{P}\left(z_{s} \rightarrow 0\right)=\lim _{\delta \rightarrow 0} \mathbf{P}\left(B_{\delta}\right)=1 .
$$

ДоКАЗАТЕЛЬСТво тЕОРЕмы 3. При каждом $s$ сделаем преобразование (15), сводя дело к случаю, когда $n=s$, а все числа $\lambda_{j}$ лежат на отрезке $[0,1]$. При этом условие (13) с учетом того факта, что $\lambda_{s} \sim$ const $s$, приобретает вид $\beta \geqslant$ const $s^{-\mu}$ с положительной постоянной const. В силу условия теоремы асимптотически при $s \rightarrow \infty$ получаем, что на отрезке $[0,1]$ имеется $s$ чисел $\lambda_{j}$ с равномерной функцией распределения. Для доказательства теоремы достаточно проверить выполнение неравенства (17). В силу третьего неравенства в (22) неравенство (17) эквивалентно (с другой постоянной) неравенству

$$
\sum_{i=1}^{s}\left(t-\beta \lambda_{i} \tau\right)^{2} \geqslant c_{1} s^{\alpha}\left(t^{2}+\tau^{2}\right), \quad(t, \tau) \in \mathbb{R}^{2},
$$


которое мы и будем проверять. Неравенство это, очевидно, выполнено при достаточно малых $|\tau| /|t|$, так что достаточно проверить его при $|\tau| /|t|>$ const $>0$, т.е., в силу однородности, при $\tau=1$. Рассмотрим минимум его левой части

$$
f(t) \equiv \sum_{i=1}^{s}\left(t-\beta \lambda_{i}\right)^{2}=s t^{2}-2 B t+C,
$$

где

$$
B=\beta \sum_{i=1}^{s} \lambda_{j}, \quad C=\beta^{2} \sum_{i=1}^{s} \lambda_{j}^{2}
$$

Минимум достигается при

$$
t=\frac{B}{s}=\frac{1}{s} \beta \sum_{i=1}^{s} \lambda_{j}, \quad|t| \leqslant \beta \leqslant \beta_{0},
$$

и равен

$$
C-\frac{B^{2}}{s}=\beta^{2} \sum_{i=1}^{s} \lambda_{j}^{2}-\frac{\beta^{2}\left(\sum_{i=1}^{s} \lambda_{j}\right)^{2}}{s}=s \beta^{2}\left(\left\langle\lambda^{2}\right\rangle-\langle\lambda\rangle^{2}\right)
$$

(здесь $\langle f\rangle=s^{-1} \sum_{j=1}^{s} f_{j}-$ усреднение по всем $\left.j=1, \ldots, s\right)$. Достаточно, таким образом, доказать неравенство

$$
s \beta^{2}\left(\left\langle\lambda^{2}\right\rangle-\langle\lambda\rangle^{2}\right) \geqslant c s^{\alpha}
$$

с некоторыми постоянными $c>0$ и $\alpha \in(0,1]$. Поскольку неравенство нужно доказать для больших $s$, для вычисления средних используем предельное (т.е. равномерное) распределение. Тогда получим

$$
\left\langle\lambda^{2}\right\rangle=\int_{0}^{1} \lambda^{2} d \lambda=\frac{1}{3}, \quad\langle\lambda\rangle=\int_{0}^{1} \lambda d \lambda=\frac{1}{2}, \quad\left\langle\lambda^{2}\right\rangle-\langle\lambda\rangle^{2}=\frac{1}{12} .
$$

Поскольку $\beta \geqslant$ const $s^{-\mu}$, требуемое неравенство выполнено с постоянной $\alpha=1-2 \mu$. Теорема доказана.

\section{СПИСОК ЦИТИРОВАННОЙ ЛИТЕРАТУРЫ}

[1] V.P. Maslov, On a Distribution in Frequency Probability Theory Corresponding to the Bose-Einstein Distribution, arXiv: math/0612394.

[2] V.P. Maslov, Negative Dimension in General and Asymptotic Topology, arXiv:math/ 0612543.

[3] В. П. Маслов, М. В. Федорюк, “Логарифмическая асимптотика быстроубывающих решений гиперболических по Петровскому уравнений”, Матем. заметки, 45:5 (1989), 50-62.

[4] В. П. Маслов, Квантовая экономика, Наука, М., 2007.

В. П. Маслов

Поступило

Московский государственный университет

10.04.2007

им. М. В. Ломоносова

E-mail: v.p.maslov@mail.ru

\section{В. Е. Назайкинский}

Институт проблем механики РАН 